\title{
Nursing Research during COVID-19 Pandemic: A Scoping Review
}

\author{
Nabeel F. Allobaney ${ }^{1}$, Abdulqadir J. Nashwan ${ }^{1,2}$, Ahmed S. Mohamed ${ }^{1}$ \\ ${ }^{1}$ Hazm Mebaireek General Hospital (HMGH), Hamad Medical Corporation (HMC), Doha, Qatar \\ ${ }^{2}$ University of Calgary, Doha, Qatar \\ Email: *allobany1@yahoo.com
}

How to cite this paper: Allobaney, N.F., Nashwan, A.J. and Mohamed, A.S. (2020) Nursing Research during COVID-19 Pandemic: A Scoping Review. Open Journal of Nursing, 10, 952-959.

https://doi.org/10.4236/ojn.2020.1010066

Received: September 24, 2020

Accepted: October 24, 2020

Published: October 27, 2020

Copyright $\odot 2020$ by author(s) and Scientific Research Publishing Inc. This work is licensed under the Creative Commons Attribution International License (CC BY 4.0).

http://creativecommons.org/licenses/by/4.0/

\begin{abstract}
Purpose: This review paper aimed to provide an overview of the published nursing research related to the COVID-19 pandemic over the last six months. Method: Online Google Scholar database was searched for COVID-19 related articles published between the 1st of Jan 2020 and 15th of June 2020. Findings: The initial search found 1930 results from combining the search between the words "nurse" and "nursing" with COVID-19. Only 48 articles met the eligibility criteria. Most of the studies focused on psychological factors (33.3\%), followed by clinical practices (29.2\%). The majority of the authors have an academic affiliation (70.8\%), only (12.5\%) have a clinical affiliation. About (21\%) of the articles were funded, (90\%) of this fund came from China. Most of the authors utilized the quantitative approach (27.1\%); the qualitative approach has been used by $(4.2 \%)$; only one article $(2.1 \%)$ utilized the mixed methodology. No study used a theoretical or conceptual framework. Mainly, the nurses conducted their research independently (52.1\%), while (35.4\%) has been done in a multidisciplinary team; $35.3 \%$ of them were done in collaboration with physicians. Conclusion: The results indicate that the nurses responded immediately to the COVID-19 pandemic by conducting researches mainly by utilizing a quantitative approach and writing a lot of editorials. To enrich the research results, nurses worked in research in collaboration with other disciplines, especially physicians. Still, the nursing research funding is under the limit. Stress and psychological factors are the primary concern for the nurses during the COVID-19 pandemic.
\end{abstract}

\section{Keywords}

COVID-19, Nursing Research, Scoping Review, Nursing

\section{Introduction}

While writing this review, the prevalence of COVID-19 cases rapidly increased, 
and more than 19 million cases have been reported and more than 700.000 confirmed deaths worldwide [1].

To respond accordingly to this pandemic, the nurses globally played a significant role in running, directing, and influencing healthcare systems to maintain it and protect it from collapse. This response takes different aspects, starting from prevention and primary care, and does not end with tertiary and critical care.

During the COVID-19 pandemic, the nurses had faced different clinical challenges like stress, anxiety, depression, and insomnia [2] [3]. Also, non-clinical challenges were addressed like alterations in healthcare policy, working hours, and psychological and social well-being [4] [5]. All these stressors might negatively alter the working environment leading to affect patient safety and quality of care.

Nurses increasingly conduct researches to adopt evidence-based practices in their careers. Cost-effective healthcare services, reduce healthcare errors, improve healthcare quality, and increase patient satisfaction, all of these aspects could be influenced by evidenced-based practices based on nursing research [6].

According to Polit and Beck, nurses have a commitment to engage in at least one role in research. "At one end of the continuum are consumers of nursing research, who read research reports or research summaries for relevant findings that might affect their practice ..., At the other end of the continuum are producers of nursing research: nurses who actively participate in generating evidence by doing research" [6].

To answer a research question, the researcher adopts the research design; this design is the researcher's overall plan to address the research question. Some of the researchers utilize the quantitative method, and others choose the qualitative approach.

In the last decade, the attention had grown in conducting mixed-method research to reach the meta-inferences. Teddlie \& Tashakkori (2009) Identified meta-inference as "a conclusion generated through an integrating of the inferences that have been obtained from the results of the qualitative and quantitative strands of an MM study" [7].

\section{Purpose}

The main purpose of this review paper was to provide an overview of the published nursing research related to COVID-19 over the last six months. The following themes were guided the review: topic, professional affiliation, collaboration with other disciplines, methods, and source of funding.

\section{Methodology}

Google Scholar was searched for articles published in the English language and carried out between January and the end of June 2020. The following keywords were included: "Nurse", "Nursing", "COVID-19", and "SARS-CoV-2". The following keyword "Nursing Home" was excluded from the search due to irrelevancy. 
Through the evaluation process, the researchers read and evaluated each article from the inclusion list; simultaneously, the data extraction template was completed. To validate the process, the included studies were reevaluated by a research assistant, and discussions and clarifications treated any discrepancies between these evaluations.

\section{Findings}

\subsection{Overview}

The initial search had 37,600 results then filtered into 48 articles after a careful assessment (Figure 1).

In this scoping review, we present the studies according to the following themes: professional affiliation, theoretical or conceptual framework, research topic, funding, study design, and collaboration with other disciplines.

\subsection{Professional Affiliation}

$100 \%$ of the authors are affiliated either with clinical (hospital) or academic institutions or both $(\mathrm{n}=48) .70 .8 \%$ of these authors have an academic affiliation $(\mathrm{n}=34), 16.6 \%$ have multiple affiliation $(\mathrm{n}=8)$, and $12.5 \%$ have a clinical affiliation $(\mathrm{n}=6)$.

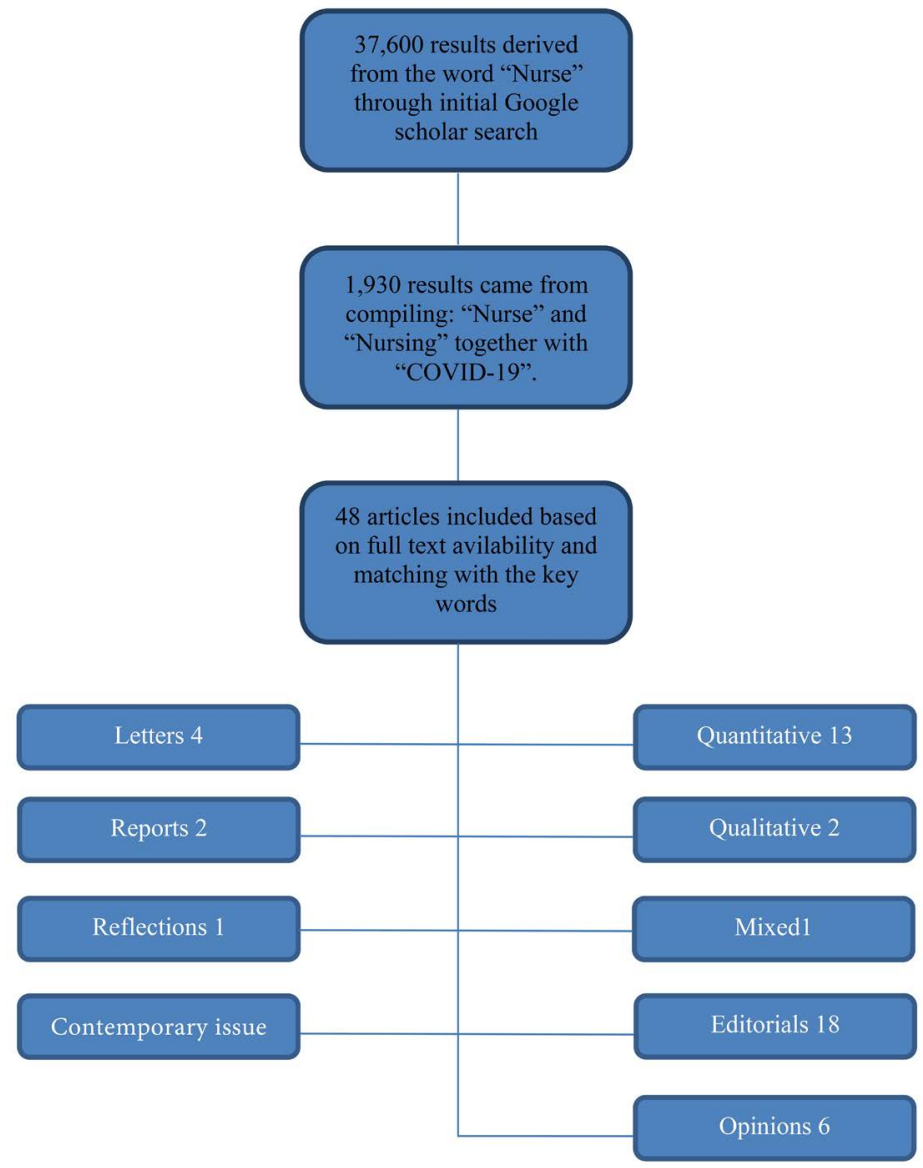

Figure 1. Selection process. 


\subsection{Theoretical or Conceptual Framework}

Out of the 48 articles included in this review, 100\% did not utilize any nursing theory or conceptual framework in their research.

\subsection{Research Topic}

Psychological factors issues focused on $33.3 \%$ of the revised articles $(n=16)$; it was the most interesting topic for nurses. Followed by the clinical practices by $29.2 \%$ of the revised articles $(n=14)$. The other aspects were the management and education with $10.4 \%$ for each ( $n=6$ for each).

One of the interesting features for this study is to track the mixed topics, where we found three articles (6.3\%) have the clinical and management focus, and two articles (4.2\%) handling the psychological and management aspects, where we also found one article (2.1\%) handling the clinical and innovation, psychological and education, management and education, one article for each.

\subsection{Funding}

The funding was reported only in $20.8 \%$ of the included studies $(n=10)$. Nine of these studies were supported by Chinese agencies like the National Natural Science Foundation of China, which funded three studies. On the other hand, only one study was funded by a USA agency.

\subsection{Study Design}

Most of the reviewed articles were editorials by 37.5\% $(\mathrm{n}=18)$. Twenty-seven-point one present $(27.1 \%)$ of the studies utilized a quantitative approach $(n=13), 4.2 \%$ of the reviewed articles utilized a qualitative approach $(n=$ $2)$, and only one study $(\mathrm{n}=1)$ utilized a mixed methodology. On the other hand, $12.5 \%$ of the studies were an opinion $(n=6), 8.3 \%$ were a letters $(n=4), 4.2 \%$ were a reports $(n=2), 2.1 \%$ was one paper as a reflection $(n=1)$, and $2.1 \%$ was one paper as a contemporary issue $(\mathrm{n}=1)$.

Obviously, the nurses responded to the COVID-19 pandemic in the first few months (from Jan 2020 till June 2020) by conduct researches address the urgent needs in psychological, clinical practices, management, and education aspects. Despite the fact that most of the articles were editorials, but it was obviously noted that the nurses invoked the quantitative methodology to get better inferences.

Furthermore, it is worthy of casting a light that the nurses in the same period didn't pay a potent effort in the qualitative approach, and that can be considered as a weakness point while the COVID-19 pandemic strikes suddenly without any preparations, so it was vital to assess the awareness and the background of the nurses accordingly by utilizing the qualitative approach.

\subsection{Collaboration with Other Disciplines}

Nursing researches during the mentioned period were broadly done by nurses $(52.1 \% ; \mathrm{n}=25)$, while $(35.4 \% ; \mathrm{n}=17)$ has been done in collaboration between 
multidisciplinary team, and $12.5 \%(\mathrm{n}=6)$ were done with collaboration with physicians including one article conducted by physicians only without nurses, but still about nurses.

\subsection{Study Participant}

Predominantly, most of the articles were editorials, opinions, reports, and letters. Only $25 \%$ of the reviewed articles included participants from clinical sites $(\mathrm{n}=$ $12)$, and $4.2 \%(\mathrm{n}=2)$ was derived from academic areas.

\section{Discussion}

\subsection{Research Approach}

There is significant growth in nursing research in the last decade [8]. In this review, the nurses responded immediately to the COVID-19 pandemic by conduct research in the early stage of this crisis.

Historically, Florence Nightingale, in the nineteenth century evolved evidence-based practice in the nursing discipline. Also, she launched the concept of improving patient outcomes by sound evidence [9].

Researches are categorized into qualitative and quantitative studies. The quantitative research hovers around the word "measure", while the qualitative research hovers around the word "perception" [10]. According to Esperon (2017), the main focus was on quantitative research, but recently, the qualitative studies have been increased as well as the mixed studies [10].

Similarly, this review supports the same conclusion as most of the included papers utilized the quantitative approach more than the qualitative or the mixed methodology.

\subsection{Importance of Nursing Education}

Several researchers emphasized the importance of clinical education for nurses [11] [12]. Nurse educators must be equipped with evidence to support frontline nurses during the COVID-19 pandemic [13]. Accordingly, most of the included studies indicated that there is an urgent need to provide the front-line nurses with the proper education, especially on donningldoffing PPE's and swabbing technique. It's vital to upskill the front-line nurses with hands-on training covering the respiratory system competencies and airway management to make them ready to fight against the pandemic, as COVID-19 mainly affects the respiratory system and increases the demand on the medical wards.

\subsection{Collaboration with Other Disciplines Acknowledgements}

Today's emerging healthcare challenges are not amenable to "isolated-islands" research approaches, where each profession is conducting their research. Building and sustaining nursing research infrastructure is considered a top priority for nursing leaders [14].

Lindqvist and colleagues (2019) highlighted the importance of collaborative 
relationships in the working environment between doctors, nurses, and clinical pharmacists [15]. Align with that, $35.4 \%$ of the revised articles were conducted with collaboration between nurses and other disciplines; this is a vital aspect that the multidisciplinary teams can provide different perspectives to enrich the research.

\subsection{Research Funding}

In the USA, the large funder for healthcare research is the federal government and institutions like the Agency for Healthcare Research and Quality (AHRQ) and the National Institutes of Health (NIH) [8]. In this review, nursing research funding still under the expectations; only $20.8 \%$ of the revised articles received funds, and most of this fund came from china. So, nursing research needs more support to accommodate the novel needs.

\section{Limitations and Recommendations}

Funding was one of the significant limitations for nurses' study by only $20.8 \%$ of the included studies received a fund. Clinical affiliation also was one of the limitations as only $12.5 \%$ of all the revised articles were done by authors who had a clinical affiliation. On the other hand, some of the authors didn't declare their education degree, making it difficult to track their education degree. The psychological and clinical aspects remained the favorite area for research.

Hands-on training and update the front-line nurses with the proper competencies will make a big difference in the patients' care. Also, provide the nurses with the proper psychological care and a safe working environment will enhance nurses' stability.

\section{Conclusion}

The results showed that the nurses responded immediately to the COVID-19 pandemic by adopting the quantitative research approach to answer the novel questions in the nursing field. The collaboration with other disciplines enriched nursing research by providing different perspectives to address the issue. We recommend to conduct more studies to address the nurse's needs during the pandemic, also to pay more effort in nursing education, especially in PPE's donning and doffing, infection control protocols, and prepare the nurses with the respiratory competencies to make them ready to fight against the pandemic. On the other hand, stress was identified as a major problem between the nurses, so we recommend conducting more studies to address this problem and how to reduce the stressors in the working environment. In the end, it is useful to cast a light over the importance of innovation during the pandemic as we are dealing with new challenges.

\section{Availability of Data and Material}

All data generated during this study are included in this published article. 


\section{Authors' Contributions}

NFA, AJN, ASM: Research design, Data collection, Literature search, Manuscript preparation.

All authors read and approved the final manuscript.

\section{Acknowledgements}

To all frontline nurses in fighting against COVID-19.

\section{Conflicts of Interest}

The authors declare no conflicts of interest regarding the publication of this paper.

\section{References}

[1] World Health Organization (2020) Coronavirus Disease (COVID-19) Weekly Epidemiological Update and Weekly Operational Update.

[2] Pappa, S., et al. (2020) Prevalence of Depression, Anxiety, and Insomnia among Healthcare Workers during the COVID-19 Pandemic: A Systematic Review and Meta-Analysis. Brain, Behavior, and Immunity, 88, 901-907. https://doi.org/10.1016/j.bbi.2020.05.026

[3] Zhuo, K., et al. (2020) Stress and Sleep: A Survey Based on Wearable Sleep Trackers among Medical and Nursing Staff in Wuhan during the COVID-19 Pandemic. General Psychiatry, 33, e100260. https://doi.org/10.1136/gpsych-2020-100260

[4] Wierenga, K.L. and Moore, S.E. (2020) Adapting to Uncertainty: Nursing Responsiveness to COVID-19. The Journal of Cardiovascular Nursing, 35, 322-323. https://doi.org/10.1097/JCN.0000000000000712

[5] Zhang, X., et al. (2020) Nurses Reports of Actual Work Hours and Preferred Work Hours per Shift among Frontline Nurses during Coronavirus Disease 2019 (COVID-19) Epidemic: A Cross-Sectional Survey. International Journal of Nursing Studies, 2020, Article ID: 103635. https://doi.org/10.1016/j.ijnurstu.2020.103635

[6] Polit, D.F. and Beck, C.T. (2008) Nursing Research: Generating and Assessing Evidence for Nursing Practice. Lippincott Williams \& Wilkins, Philadelphia.

[7] Tashakkori, A. (2009) Are We There Yet? The State of the Mixed Methods Community. SAGE Publications, Los Angeles. https://doi.org/10.1177/1558689809346151

[8] Munhall, P. (2012) Nursing Research. Jones \& Bartlett Learning, Burlington.

[9] Mackey, A. and Bassendowski, S. (2017) The History of Evidence-Based Practice in Nursing Education and Practice. Journal of Professional Nursing, 33, 51-55. https://doi.org/10.1016/j.profnurs.2016.05.009

[10] Esperón, J.M.T. (2017) Quantitative Research in Nursing Science. Escola Anna Nery Revista de Enfermagem, 21, 1-2. https://doi.org/10.5935/1414-8145.20170027

[11] Cheraghi, R., Namadi, F. and Jasemi, M. (2019) Effectiveness of the Clinical Teaching Associate Model in Clinical Nursing Education. Nursing and Midwifery Studies, 8, 132-136. https://doi.org/10.4103/nms.nms_7_18

[12] Farzi, S., Shahriari, M. and Farzi, S. (2018) Exploring the Challenges of Clinical Education in Nursing and Strategies to Improve It: A Qualitative Study. Journal of Education and Health Promotion, 7, 115. 
[13] Nashwan, A.J., Mohamed, A.S. and Kelly, D.R. (2020) Nursing Education in the Emergence of COVID-19. Open Journal of Nursing, 10, 595-597. https://doi.org/10.4236/ojn.2020.106040

[14] Nashwan, A.J., et al. (2017) Development of Nursing Research in Qatar: 15-Year Status Report. Open Journal of Nursing, 7, 242-252.

https://doi.org/10.4236/ojn.2017.72020

[15] Lindqvist, M.H., Gustafsson, M. and Gallego, G. (2019) Exploring Physicians, Nurses and Ward-Based Pharmacists Working Relationships in a Swedish Inpatient Setting: A Mixed Methods Study. International Journal of Clinical Pharmacy, 41, 728-733. https://doi.org/10.1007/s11096-019-00812-8 